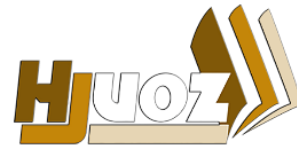

hjuoz.uoz.edu.krd p-ISSN: 2410-7557
كَّوارا زانستيّن مروّقايهتى يا زانكوّيا زاخوّ

مجلة العلوم الانسانية لجامعة زاخو

Humanities Journal of University of Zakho (HJUOZ)

Vol. 6, No. 2, pp. 485-494, Jun-2018

\title{
موقف المملكة العربية السعودية من قيام الثورة الإسلامية الإيرانية عام 1979
}

\author{
ناظم يونس عثمان 1* و نزار زهير شفيق2

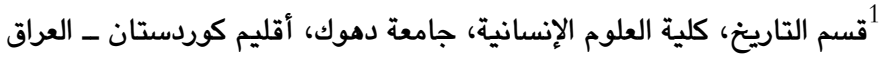

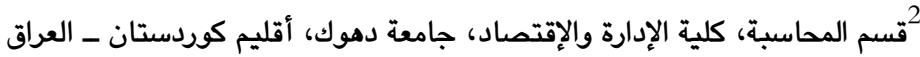

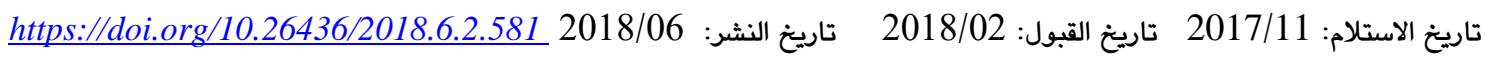

الملخص:

إحتلت المملكة العربية السعودية مكانة بارزة على الصعيدين الأقليمي والدولي لما امتازت به من ثقل ديني وسياسي وإقتصادي، وقد

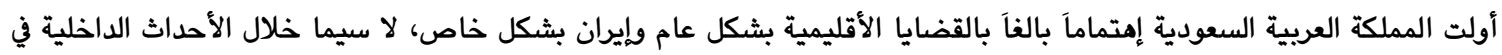

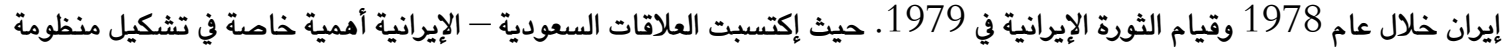
العلاقات الإيرانية مع الدول الخليجية، وذلك بحكم الثقل السياسي والتاريخي والديني والإقتصادي الذي تمثله الدولتان.

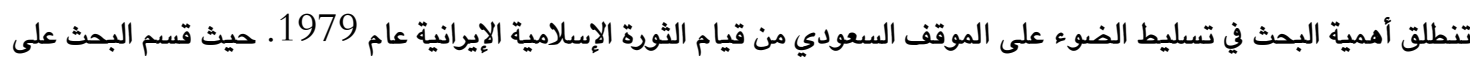
أربعة محاور، وجاء في مقدمة البحث التمهيد لإلقاء نظرة في العلاقات السعودية - الإيرانية قبل عام الإئ 1979، وجاء التعاء المحور الأول ليسلط

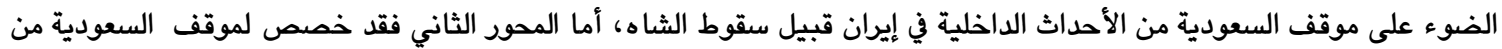
قيام الثورة الإيرانية، أما المحور الثالث فقد بحث عن موقف المملكة العربية السعودية من مبدأ تصدير الثورة إلى الخارج، وركز المحور الرابع لتطور العلاقات بين البلدين بعد إحتلال السفارة الأمريكية في طهران. الكلمات الدالة: المملكة العربية السعودية، الثورة الاسلامية، ايران.

شهدت العلاقات السعودية - الإيرانية تحسناً واضحاً في كافة المجالات، بعد تأسيس المملكة العربية السعودية عام 1932 حتى سقوط الشاه محمد رضا البهلوي عام 1979، حيث مثلت فترة حكم عبدالعزيز آل

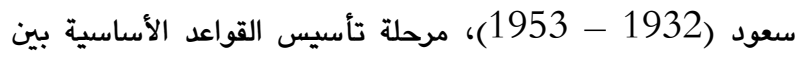
البلدين، والبحث في الوسائل والسبل اللازمة لتطويرها. وإستمرت العلاقات بين البلدين على هذا المنوال في فترة الحكم الملك سعود بن

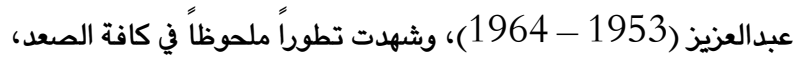
ولا سيما في الفترات اللاحقة خلال عهد الملك فيصل بن عبدالعزيز

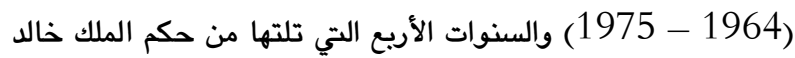
بن عبدالعزيز حتى سقوط الشاه عام 1979، حيث إتسمت العلاقات بين البلدين الإستقرار والإزدهار، بسبب المتغييرات التي شهدتها المنطقة، والتي دفعت بالبلدين الى التعاون والإلتقاء في تأمين الإستقرار،

وحماية مصالحها المشتركة خدمة لأهدافهما الإستراتيجية (4). لقد حرصت كل من المملكة العربية السعودية وإيران قبل عام 1979، المحافظة على العلاقات الإستراتيجية بينهما، من خلال إقامة العلاقات

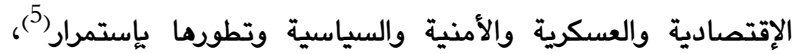
والسعي دائماً نحو تنسيق السياسة الخارجية بين البلدين، خاصةً فيما يخص القضايا الأقليمية ويما يخدم المصالح المشتركة بينهما، فضلاً

\section{1.تمهيد}

تعود إقامة العلاقات الدبلوماسية بين السعودية وإيران إلى عام 1927، عندما عينت إيران حبيب الله خان هويدا كأول دبلوماسي لها في منطقة نجد والحجاز، وشهدت العلاقات بين البلدين بعدها تطوراً ملحوظاً وصل الى مرحلة التوقيع على معاهدة الصداقة بين البلدين عام 1929، تضمنت وضع القواعد الأساسية للتعاون السياسي والدبلوماسي والتجاري بينهما، وعلى خلفية هذه المعاهدة تبادل رضا شاه البهلوي (1878 - 1944) والملك عبدالعزيز بن عبدالرحمن آل سعود (1876 - 1953)، برقيات التهنئة بهذه المناسبة(1). وبعد أن تمكن عبدالعزيز آل سعود من توحيد أقاليم نجد والحجاز وملحقاتها، وإعلان تأسيس المملكة العربية السعودية في أيلول عام 1932، إعترفت إيران بها كخطوة أولى نحو تعزيز علاقتها مع المملكة الجديدة، وإستمرت العلاقات تسير وفق هذا الإتجاه حتى عام 1941 (2). بعد إحتلال إيران من قبل القوات السوفيتية والبريطانية، وتنازل رضا شاه عن العرش لإلإبنه محمد رضا البهلوي(3). 
حيث أعتقدت الحكومة السعودية، بأن جزء كبير من الإضطرابات

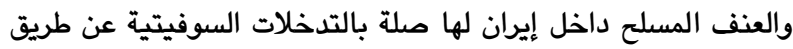

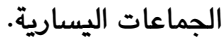

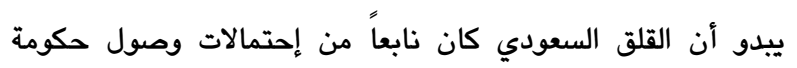

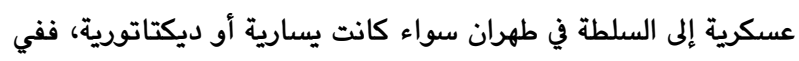
الحالة الأولى يمكن أن يتسرب تيار اليسار إلى مجتمعات الخليج. وفي

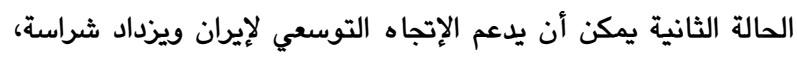

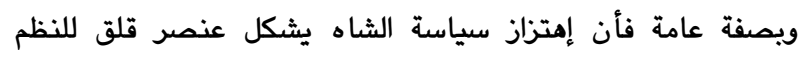

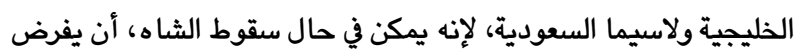

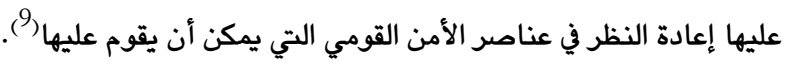

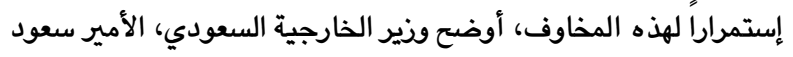

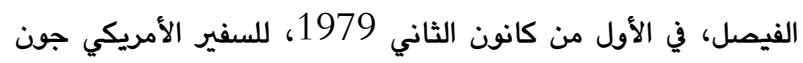

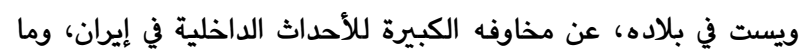
ستؤل إليه المجريات الأقليمية في حال سقوط النظام الإيراني، مؤكداً

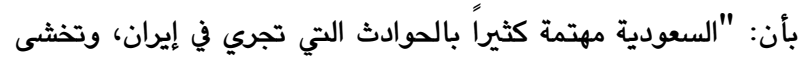
الحكومة السعودية إنه في حال رحيل الشاه فأن كل معالم الوحدة

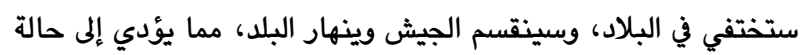
من الفوضى والغموض لن تكون جيدة للمنطقة... ان الشاه بحاجة إلى

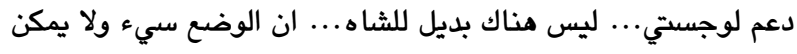

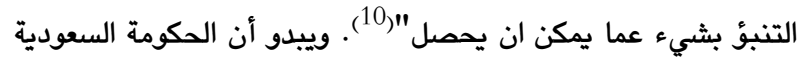
كانت لديها قناعة تامة بأن رحيل الشاه وتغيير النظام سيكون له انه مردودات سلبية على المملكة العربية السعودية ومنطقة الخليج.

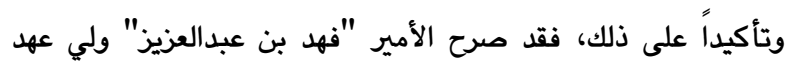

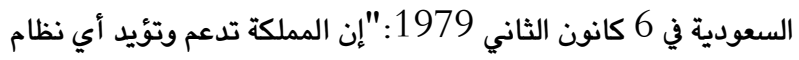

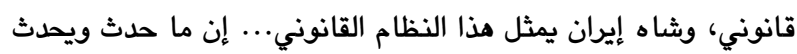
في العاصمة الإيرانية وغيرها من مدن وقرى إيران هو في نظر المملكة العربية السعودية مسألة داخلية تخص الإيرانيين وحدهم، وأن يضع مدرن الإيرانيه الإيرانيون بأنفسهم نهاية لهذا الذي يحدث بينهم... إن مما لا شك فيه

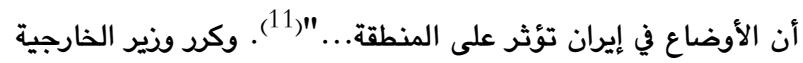

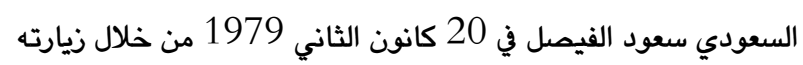

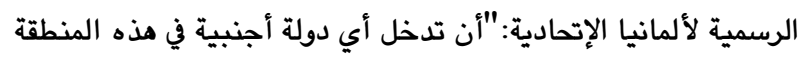

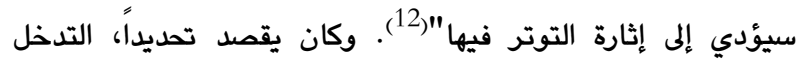
السوفيتي في الأوضاع الداخلية في إيران والخليج العربي.

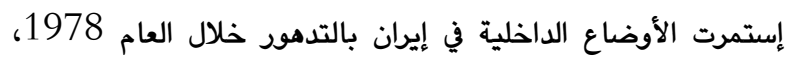

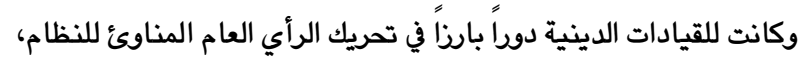
وقيادة المظاهرات ولا سيما في المناطق النفطية في الجنوب، مما أثرت

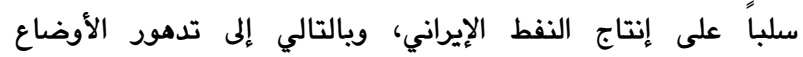
الإقتصادية، وزيادة التظاهرات الشعبية في معظم المدن الإيرانية

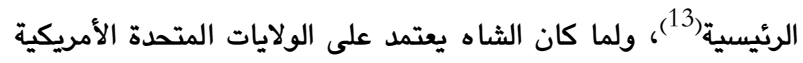

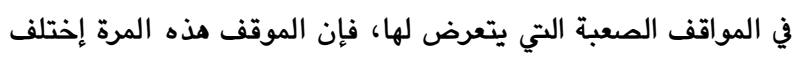

عن تسوية الخلافات والتحديات التي إعترضت هذه العلاقات بشكل سلمي، والحيلولة دون إنعكاس تلك التحديات عل مسار العلاقات الثنائية بينهما، والمحافظة على إستقرارها وديمومتها قدر الإمكان.

\section{2. موقف السعودية من الأحداث الداخلية في إيران قبيل سقوط الشاه}

بدأت الأوضاع الداخلية في إيران تشهـ إضطرابات واسعة منذ أواخر

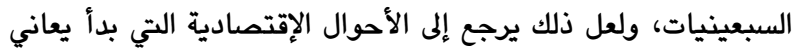
منها الشعب الإيراني، نظرا لإنصراف الشاه محمد رضا الى دعم القدرات العسكرية للجيش الإيراني على حساب التنمية الإقتصادية داخل البلاد، حيث كان الشاه ينفق على مشروعات الدفاع وشراء الأسلحة من الإنى الولايات المتحدة ما يعادل ثلث الميزانية العامة لإيران، التي بدأت تعاني من العز المالي منذ عام 1976، على الرغم من ثروتها البترولية

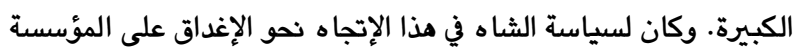
العسكرية، ومنح الولايات المتحدة الأمريكية العديد من الإمتيازات في

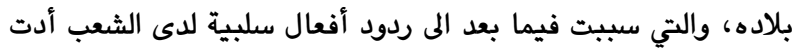

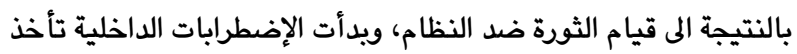

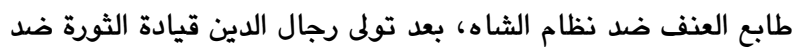
الشاه ونظامه إبتداءاً من شباط 1978، 1978، والتي إستمرت طوال العام

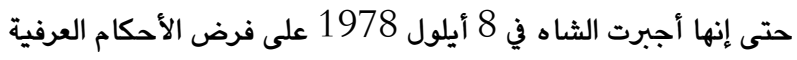

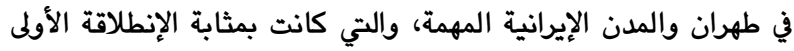

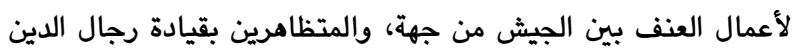
من جهة أخرى. والتي تضامنت معها فيما بعد معظم الأحزاب السرية

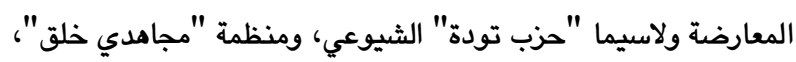

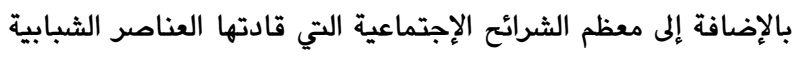

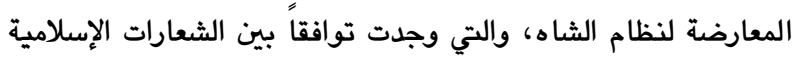

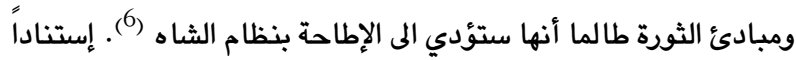
إلى ميراث التنافس بين البلدين حول حماية أمن الخليج من التهديدات اليسارية الداخلية، وتهديدات التغلغل السوفيتي في المنطقة، وكذلك الخطابات المتكرة لشاه إيران حول قدرة بلاده على حماية أمن

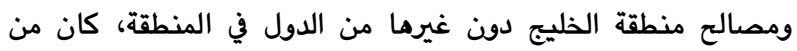

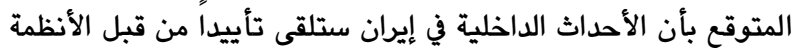

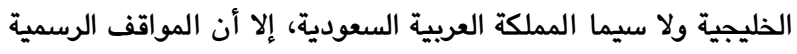
لهذه البلدان تجنبت مذا الإحتمال، بل شاركت النظام في مساعية

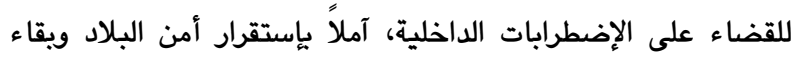

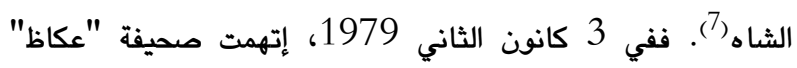
السعودية الرسمية حكومة الإتحاد السوفيتي بالعمل على خلق التوتر في

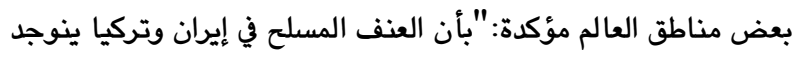
بدود الإتحاد السوفيتي ويعكس إصراره على السعي إلى خلق بؤر للتوتر،

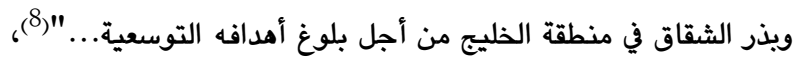


في تحسين العلاقات مع جميع دول الخليج العربي، والتخلي عن سياسات الثاه السابقة في أداء دود "ثرطي الخليج"، وتبني سياسة جديدة ترضى جميع دول المنطقة، وإبعاد التدخلات الأجنبية في المنطقة. ويناءاً عليه، ألغت الحكومة الإيرانية الجديدة في آذار 1979، الصفقة الصفال

التسليحية السابقة التي عقدتها مع الولايات المتحدة الأمريكية (24). ولعل هذا التوجه الجديد من الحكومة الإيرانية برئاسة بازركان، قد ثجع الملك خالد بن عبدالعزيز على إرسال برقية تهنئة إلى آية الله باله الخميني في 3 نيسان 1979، بعد يومين من إعلان قيام جمهورية إيران إنان الإسلامية في الأول نيسان عام 1979، مؤكداً فيها عن سعادته ومباركته لقيام نظام جديد مبني على أسس الإسلام، بقوله :"... فإنه ليسرني بمناسبة إعلان قيام الجمهورية الإسلامية في إيران أن أعرب لسماحتكم عن صادق تهنئتي القلبية. ولا ثك بأنه كان لهذا الإعلان أحسن الأصداء في المملكة العربية السعودية التي تلتزم بالشريعة الإسلامية عقيدة ومنهجاً وتطبيقا، وأنه لمن دواعي السرود والغبطة حقاً أن تقوم هذه الجمهورية الناشئة على الدعائم الإسلامية الوطيدة، وأن

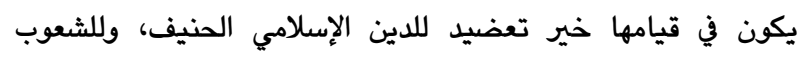
الإسلامية التي تتطلع إلى ما فيه خيرها وعزها وكرامتها، وأرجو أن يجعلكم الله في مقدمة العاملين على نصرة الإسلام والمسلمين في كل مكان، كما أتمنى للشعب الإيراني الشقيق في ظل الشريعة الإسلامية كل

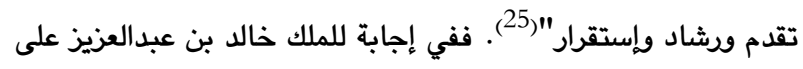

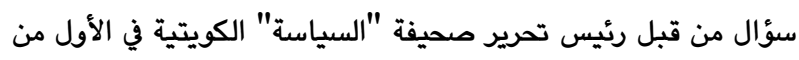
تموز 1979، عن طبيعة العلاقات بين المملكة والحكومة الجديدة في إيران، قال:"كما هو معروف فإننا نرحب دائماً بقيام الحكم الإسلامي في أي بلاد، ونحن نعتقد أن إقامته في إيران مدعاة إلى المزيد من التقارب والتفاهم، ومذا ينسجم مع دعوتنا الدائمة إلى إتخاذ الشريعة الإسلامية قاعدة للحكم"(26). إلاّ أن تطبيع العلاقات بين البلدين لم تدم طويلاً، حيث بدأت الشكوك تظهر لدى الجانب السعودي حول توجهات السلطة الجديدة في إيران في تصريحات مسؤوليها حول مبدأ تصدير الثورة، والأفكار المذهبية والطائفية التي إتسمت بها طبيعة النظام الجديد، وحاولت الحكومة السعودية جاهدة في عدم التصادم مع القيادة الجديدة في إيران، بل التكييف مع الواقع الجديد والإصطفاف معها للحيلولة دون توسع النفوذ السوفيتية في منطقة الخليج العربي.

\section{4. موقف السعودية من مبدأ تصدير الثورة إلى الخارج}

اتسمت العلاقات السعودية - الإيرانية، منذ البداية، بحالة من الترقب والحذر الشديد لحين توضيح نوايا وأفعال النظام الجديد في إيران، ويدأت المملكة العربية السعودية تنتظر ما ستؤول إليه النتائج من تطور الأحداث في الداخل الإيراني، وما ستتبعه القيادة الجديدة من أدوار سياسية في إدارة ملفها الخارجي وتعاملها مع الدول الأقليمية، ولا سيما
ريما للضغوط الداخلية والدولية، حيث أعلن الرئيس الأمريكي "جيمي كارتر - Jimmy Carter" (1977-1981) أن الولايات المتحدة لن تتدخل في الشؤون الداخلية في إيران(14). وهكذا إجتمعت الأسباب، التي أدت إلى الإضطرابات العنيفة في أنحاء

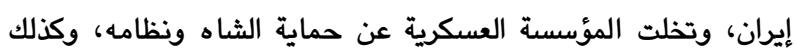
تخلت الولايات المتحدة عن حليفها الذي سبق لها أن أنقذته أيام ثورة مصدق عام 1953(15)، فلم يجد الشاه مفراً في كانون الثاني 1979 إلآ أن يعين شاهبور بختيار(16) رئيساً للوزراء، المعروف بميوله المعتدلة، في محاولة يائسة منه للخروج من الأزمة، في الوقت الذي أعد بلدي الشاه نفسه للتخلي عن السلطة إلى مجلس الوصاية على العرش

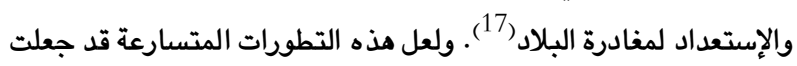
الولايات المتحدة حليفة الشاه تعلن تأييدها لقراره بمغادرة إيران، وفي

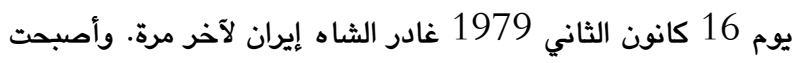
الفرصة مهيأة للعناصر الدينية والأحزاب المعارضة والشعب الإيراني لإستقبال آية الله الخميني (1902-1989) ، الذي عاد إلى طهران في

الأول شباط 1979، ليتسلم رجال الدين زمام الحكم في إيران(18).

\section{3. موقف السعودية من قيام الثورة الإيرانية}

كانت المملكة العربية السعودية، خلال الأحداث الداخلية في إيران، تراقب الموقف عن كثب حرصاً منها على عدم إنتشار الثورة في الخارج، والسعي على إبقاء إستقرار الوضع في المنطقة، ورغبة منها في الحفاظ

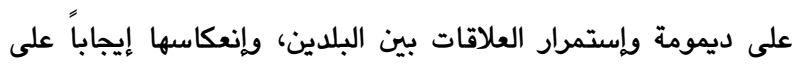
منطقة الخليج ودولها(19). ويبدو أن الحكومة السعودية لم تولى إمتماماً للأحداث الداخلية في إيران، إلا بعد أن توضحت الصورة لديها بأن نظام شاه بدأ يفقد توازنه ولاسبيل أمامه إلاً الرحيل وتسليم السلطة إلى إلى

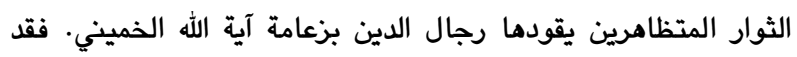
أعلنت المملكة العربية السعودية ترحيبها بعودة آية الله الخميني إلى بلى بلهين إيران، وإعترفت بتشكيل الحكومة الإيرانية المؤقتة برئاسة مهدي

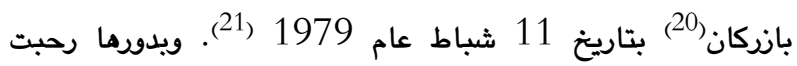
الحكومة الإيرانية الجديدة بتحسن العلاقات مع المملكة العربية السعودية، وأكدت على حرصها في تمتين العلاقات معها ومع دول الخليج بصفة خاصة، والدول العربية بصفة عامة(22)، وأزداد التقارب الإيراني - العربي بسبب موقف الحكومة الإيرانية الداعم للقضية الفلسطينية، وقرارما بقطع العلاقات الدبلوماسية مع دولة إسرائيل في 19 شباط .23)1979

ويعد إستلام مهدي بازركان لرئاسة الحكومة في إيران، أرسلت الحكومة السعودية برقية تهنئة لمناسبة توليه المنصب الجديد، دعت فيها إلى تعزيز العلاقات بين البلدين على أساس حسن الجوار وعدم التدخل في الشؤون الداخلية. ويدورها أعلنت الحكومة الإيرانية الجديدة عن رغبتها 
إيجابية بين البلدين(34). ومن جانبها، قامت الحكومة السعودية بإرسال (500) رجل من قوات الحرس الوطني إلى البحرين في 14 أيلول 1979 بشكل سري، لمساعدة حكومة البحرين للحفاظ على النظام في وجه

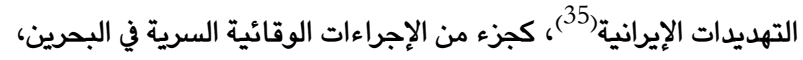
إستعداداً لمواجهة أي إنتفاضة أوتدخل إيراني في البحرين. ومن جانب آخر، تطرق ولي العهد الأمير فهد بن عبدالعزيز خلال لقائه

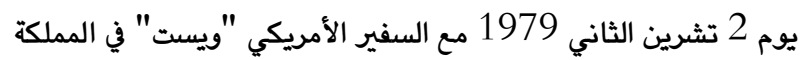
العربية السعودية، حول الأوضاع الداخلية في إيران وإنعكاسها على دول الخليج العربي والمنطقة، مشيراً إلى أن "التطورات الأخيرة في إيران أثبتت عدم مبالاة الإدارة الأمريكية وعجزها في تقديم البدائل على إلى الحئ

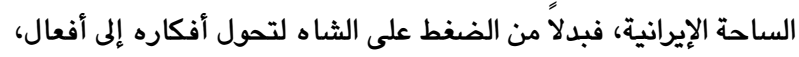

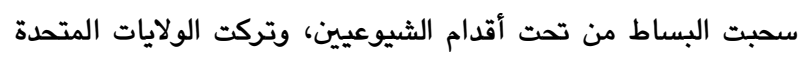
الشاه يرحل (36)، وأشار أيضاً إلى الفوضى الداخلية في إيران وسيطرة

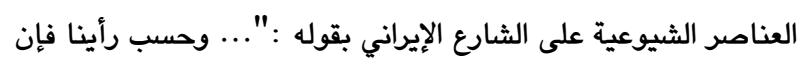

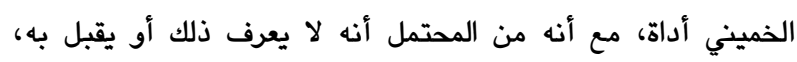

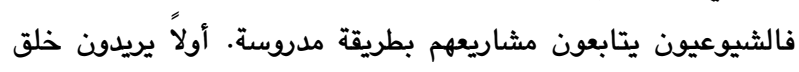

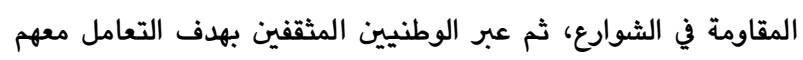
بالتدرج ويفعالية أكثر... إن هذه الأعمال البربرية لا صلة لها بالإسلام الذي ترفرف رايته بشكل مصطنع في إيران"(37)، وأشار ولي العهد العدال السعودي أيضاً:"بأن إيران تهدد البحرين والكويت والدول العربية الأخرى في الخليج، ولم ترد أي كلمة تحذير من الرئيس كارتر لإيران، لطمأنة، ليس فقط الدول الضعيفة كالبحرين، بل أيضاً أصدقاء أمريكا

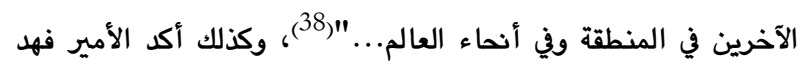
للسفير الأمريكي:"كفى ثم كفى، نحن وكل الشعوب الضعيفة في العالم لا نستطيع الجلوس بخمول ونترقب شريعة ألغاب تسود. أولاً أثيوبيا،

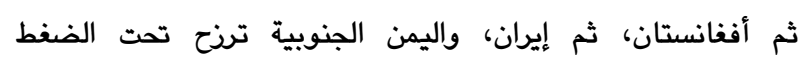
السوفياتي...." (39).

ويبدو مما سبق، بأن الحكومة السعودية كانت تتخوف من سيطرة الشيوعية على السلطة في إيران أكثر من التيار الديني بقيادة آية الله

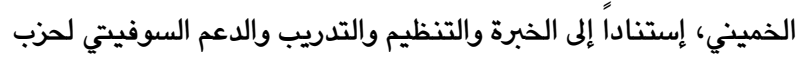
"تودة" الشيوعي وعناصره في إدارة الأزمة الداخلية في إيران، وكذلك إلك عدم قدرة القيادات الدينية في إدارة الدولة لفترة طويلة لإنعدام الخبرة لديها، وكذلك تعدد مصادر القرار، وعدم وجود رؤية سياسية واضحة

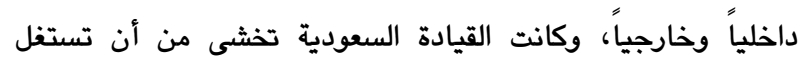
الأوضاع الداخلية في إيران من قبل الإتحاد السوفيتي. أن أحداث المنطقة والمتغييرات الجديدة، قد وضعت صانعي القرار في مإن السعودية أمام موقف صلب حيال السياسة الأمريكية في المنطقة، بسبب إختلاف وجهات النظر حول بعض القضايا الإقليمية، أهمها: الوضع في إيران وسقوط الشاه، وتلكؤها في دعم نظامه، مما يؤكد عدم قدرتها على ضمان بقاء أي نظام حليف عندما يصل إلى حافة السقوط،
الدول الخليجية، وكانت تسعى دوماً للحفاظ على العلاقات الطبيعية والمتميزة مع النظام الإسلامي الجديد في إيران(27). وتدريجياً بدأت الصحافة الإيرانية، وتصريحات بعض القيادات الثورة

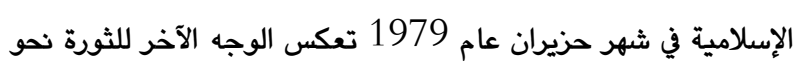
مطامعها حول الخليج العربي وأنظمتها، حيث أخذت تطالب بجزيرة البحرين وضمها إلى الأراضي الإيرانية، وكذلك تهدد بتصدير الثورة(28)

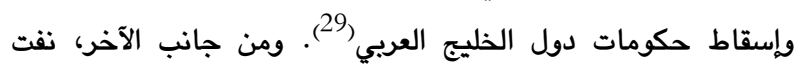
الحكومة الإيرانية فيما بعد تلك التصريحات بشآن طموحاتها وسياسة دولة

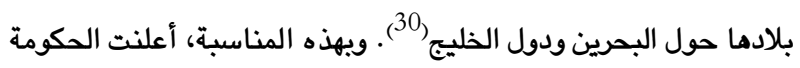

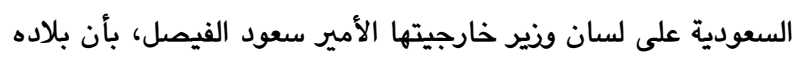
تبدي إرتياحها من إعلان الحكومة الإيرانية نفيها لما صدر عن أحد

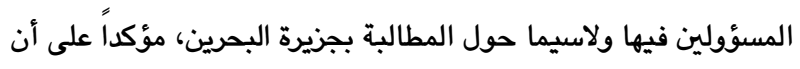
"التصريحات الإيرانية الإخيرة ونفي حكومتها نفياً قاطعاً حول مطالبتها بدولة البحرين، نحن نقدر كل التقدير النفي القاطع والصريح من قبل الحكومة الإيرانية لكل ما ورد من تصريحات من قبل أطراف غير نعري مسؤولة..." المكومة الإيرانية وفي مقابلة أخرى أجراها رئيس تحرير مجلة "الحوادث" اللبنانية مع ولي العهد السعودي الأمير فهد بن عبدالعزيز في 10 كانون الثاني

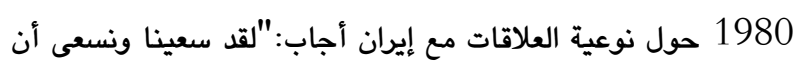
تكون علاقات جيدة، نحن نعيش في منطقة واحدة مع إيران ولا مصلحة لنا ولا لها أن يكون هناك سوء تفاهم بيننا، لا سيما وأن الحكم الجديد في إيران يعمل تحت شعار العقيدة الإسلامية، وهو شعارنا في المملكة

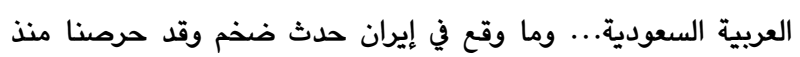

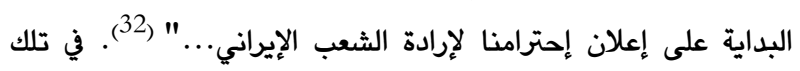
الفترة، أرادت السعودية الإعتراف بالواقع الجديد في إيران، مهما يكون إلهاري نظامهم، لاسيما إذا كان ليس لديهم الصلة بالشيوعية اليسارية والإتحاد السوفيتي. شهدت العلاقات الرسمية بين المملكة العربية السعودية وإيران في تلك

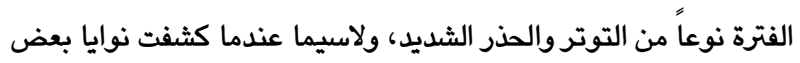
المسؤولين الإيرانيين حول المطالبة بالبحرين، والتصريحات التهديدية لأمن دول الخليج العربي، مما أدى إلى بداية تحفظ المملكة العربية السعودية في موقفها تجاه الجمهورية الإسلامية في إيران، حيث رأت في التصريحات الإيرانية ما يثي القلق، وأن إيران أصبحت قوة ثورية تهدد منطقة الخليج. شعرت حكومة مهدي بازركان المعتدلة، بالقلق حول

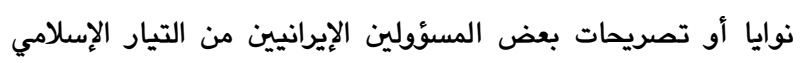

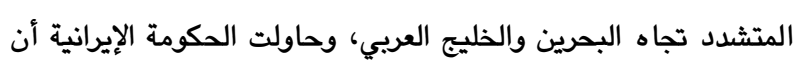
تطمئن الحكومة السعودية حول تلك التصريحات، وكلفت وزير خارجيتها إبراهيم يزدي بتوجه دعوة رسمية الى وزير الخارجية السعودي في 5 آب 1979 بزيارة إيران للتباحث حول أهم المستجدات

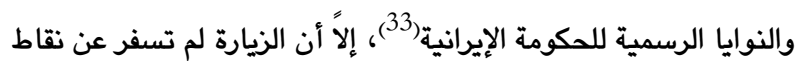


ومن هنا، بدأت الحكومة الإيرانية الجديدة، بشن حملة إعلامية معادية

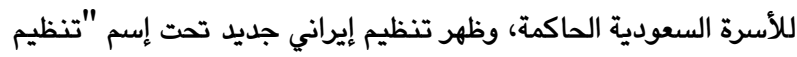
الثورة الإسلامية في شبه الجزيرة العربية" بهدف التدخل وإثارة الفتنة في منطقة الخليج برمتها. وكذالك صرح الخميني بأنه يتكلم بإسم كل

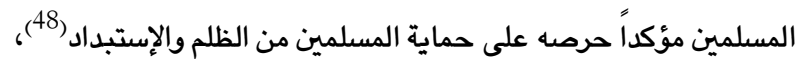
لاسيما بعد أحداث المسجد الحرام في مكة المكرمة في تشرين الثاني

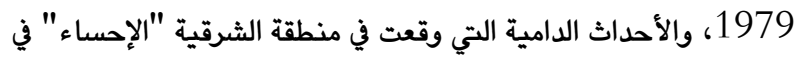

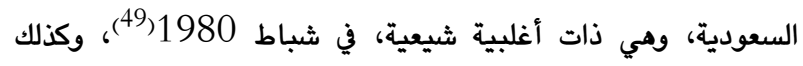
التصريحات الإيرانية المستمرة حول ضم دولة البحرين إليها. لم يكن

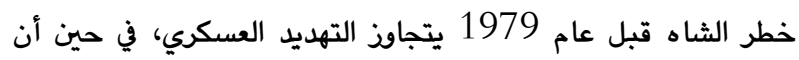
الثورة الإيرانية الإسلامية كانت تحمل إضافة إلى التهديد العسكري تهديدا

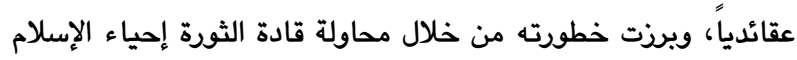

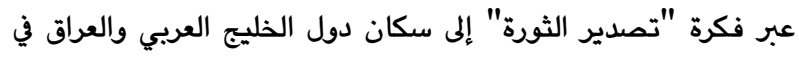
المناطق ذات الأغلبية "الشيعية"، ولم تكن النخب الحاكمة في دول الخليج بعيدة عن هذا الهجوم، فقد إعتبر الإمام الخميني النظم الخليجية الخداف

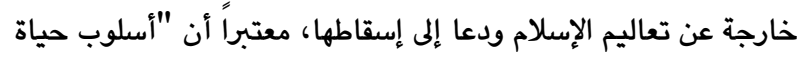

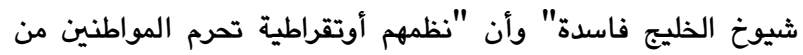

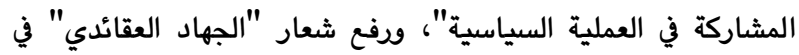

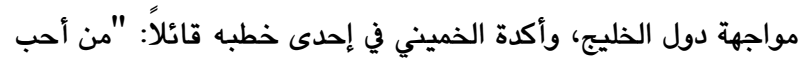

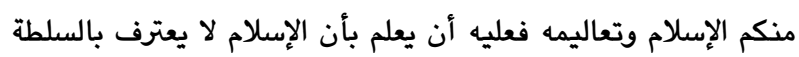
الوراثية ويالخلافة الملكية" (50. وقامت السلطات الإيرانية بإطلاق تصريحات مناوئه للحكومة السعودية بعد حادثة إحتلال مجموعة مسلحة للحرم المكي الشريف في تشرين الثاني 1979، ويدأت الإذاعة الإيرانية تتهجم على السعودية، وتتهمها بالإلحاد ومعادات الإسلام، لا بل دعمت حكومة طهران المنظمات

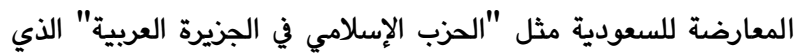

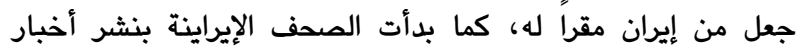
الإضطرابات والتظاهرات التي تحدث في المملكة العربية السعودية ويتحريص من جماعات تمولها الحكومة الإيرانية(51). ويدأت الأحداث تتسارع في المنطقة بعد زيادة حدة التوترات بين الحكومة الأيرانية والعراقية، التي أدت بالنتيجة الى تدهور العلاقات

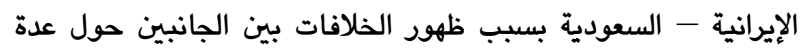

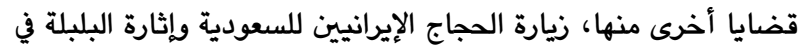
مناسك الحج، والخلافات المتزايدة بين الطرفين داخل منظمة أويك النفطية حول أسعار النفط والحصص الإنتاجية لدول الأعضاء

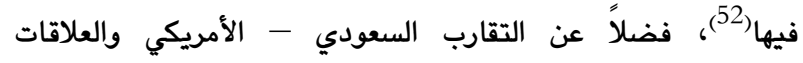
الأستراتيجية بين الطرفين، التي بدأت تتزايد بشكل فعلي ومنظور، حيث إستقبلت المملكة العربية السعودية عدداً من الطائرات الأمريكية

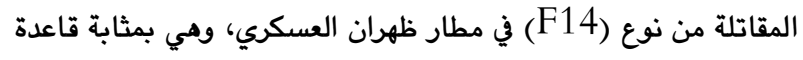

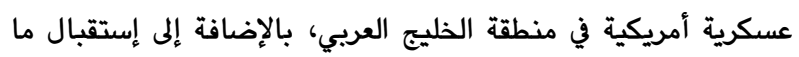

بل قد تساهم هي في إسقاطه عندما تفقد الثقة في قدرته على ضمان المصالح الأمريكية، أو عندما يعتمد سياسة قد تتقاطع مع السياسة الأمريكية. حيث أدركت القيادة السعودية أن الموقف الأمريكي من شاه

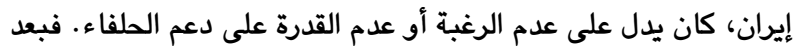

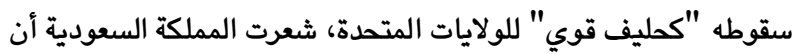

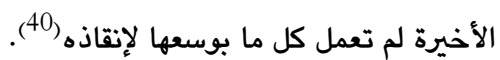
كانت الإستراتيجية السعودية، في كل الأحوال، تستهدف الحفاظ على الوضع الراهن في المنطقة والوقوف بوجه التجاوزات الإيرانية، ومحاولة إحتوائها، من خلال تطبيق مبدأ التفاهم وتسوية الخلافات مع إيران الإنانية وماركان بالطرق الدبلوماسية، ويسياسة النفس الطويل والإبتعاد عن المهاترات

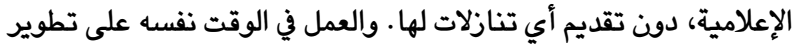

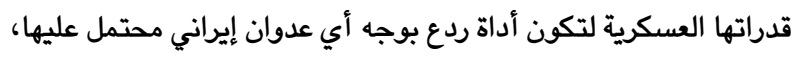
أو على دول الخليج إلى جانب دبلوماسيتها الهادئة (41).

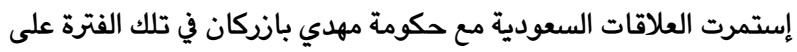
الرغم من حدوث بعض التوترات والتشنجات بين الجانبين، لاسيما إختلاف وجهات النظر حول الأحلاف الدولية، ودود الولايات المتحدة الأمريكية في المنطقة، وعلاقاتها الإستراتيجية مع المملكة العربية السعودية، لاسيما مواقفهما المشتركة حول بعض القضايا المتعلقة

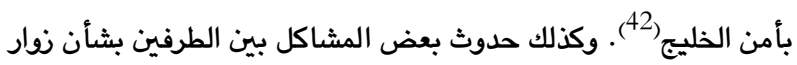

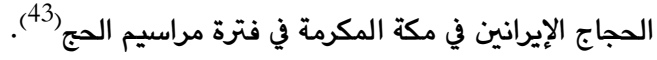
5. تطور العلاقات بين البلدين بعد إحتلال السفارة الأمريكية

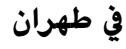

تطورت الأحداث الداخلية بشكل متسارع وألقت بخلالها على العلاقات السعودية الإيرانية، وكانت مصادر القرار داخل السلطة الإيرانية متعددة ومختلفة، عبَرت عن إختلاف نوايا رجال الثورة والسلطة في إيران، فسقطت حكومة مهدي بازركان المعتدلة عقب قيام مجموعة من

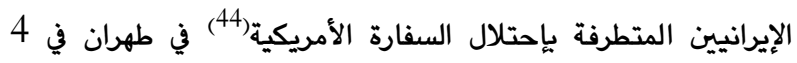

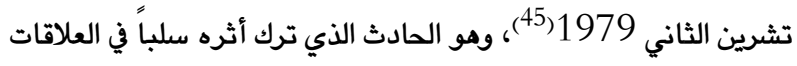

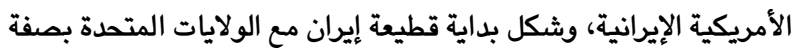
خاصة والغرب بصفة عامة (46).

شجبت المملكة السعودية اقدام الثوار الإيرانيين بإحتلال السفارة الأمريكية في طهران، وكان موقف السعودية من هذه الأزمة هو شجبها لقيام إيران بإحتلال السفارة الأمريكية في طهران، على أساس أن ذلك الك فهردان

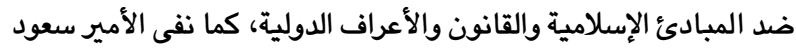
الفيصل وزير الخارجية السعودي آنذاك، قيام بلاده بالتوسط بين الولايات المتحدة وإيران بشأن حل أزمة الرهائن(47). وأتخذت الحكومة بالتهاية

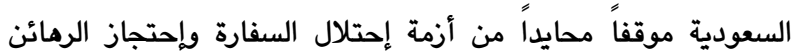
الأمريكان ولم تبادر إلى التوسط بين الطرفين، أو التدخل لحل الأزمة لخطورة الموقف وحساسيته. 
الإسلامية "ظاهريا" لحين إستتباب الأمور الداخلية، وبيان موقف القيادة الجديدة في نهجها على الصعيدين الداخلي والخارجي، ولا سيما موقفها من الدول الأقليمية والخليجية، وهذا التأييد الظاهري للثورة الإسلامية كان سلوكاً منها في سياستها الخارجية تجاه الأحداث الإيرانية، ولهذا أرادت من تأييدها الظاهري أن يكون موقفاً إيجابياً منها

تجاه الثورة الجديدة.

حاولت الحكومة السعودية جاداً أن تتعامل مع القيادة الإيرانية الجديدة بحذر شديد للحيلولة دون الإصطدام معها أو خلق مشاكل معها في منطقة الخليج، بسبب مؤشرات أفكارها المذهبية وآيدولوجيتها للثورة، بل حاولت السعودية منذ البداية إستثمار التوجهات الجديدة للنظام الإسلامي المعادية للنفوذ السوفيتي في المنطقة من أجل إبعاد الأخيرة من منطقة الخليج العربي، إلاً أن سرعان ما ظهرتيني فئول النوايا الإيرانية الجديدة حول المطالبة بالبحرين، وتهديدها لبعض الدول الخليجية الأخرى بعد إستلام السلطة بفترة وجيزة، مما دفعت بالحكومة السعودية بلدية إلى التعامل مع النظام الجديد بتحفظ شديد والتعامل معها بحذر طبقاً للمواقفها وأفعالها الجديدة.

\section{7. الهوامش}

1. للمزيد من المعلومات أنظر: د. محمد عبدالرحمن يونس عيدان، العلاقات الإيرانية - السعودية 1941 - 1979 (دراسة تأريخية سياسية)، مجلة دراسات إقليمية، جامعة الموصل، السنة (4)، العدد (7)، كانون الثاني 2007، ص 190.

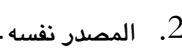
3. م.م. حيدر عبدالواحد الحميداوي، مصالح بريطانيا الإقتصادية في إيران في عهد رضا شاه بهلوي، مجلة دراسات إيرانية، جامعة البصرة، العدد (8-9)، 2008،

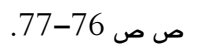
4. للمزيد من المعلومات حول هذا الموضوع أنظر: د. عبدالحكيم عامر الطحاوي، العلاقات السعودية - الإيرانية وأثرها في دول الخليج العربي 1951 - 1981، الطبعة الأولى، مكتبة العبيكان، الرياض 2004، ص 25 وما بعدها، د.تاج السرحران، إ. محمود حسن أحمد، السياسة الخارجية في عهد الملك فيصل بن عبدالعزيز آل سعود، الدار العربية للموسوعات، الطبعة الأولى، بيروت 2008، ص ص 90 - 97 “ د.عصام السيد عبدالحميد، العلاقات السعودية الإيرانية 1982 ـ 1997، الطبعة الأولى، عين للدراسات والبحوث الإنسانية والإجتماعية، القاهرة

$$
\text { 2006، ص } 15 \text { و ويعدها. - - 206 }
$$

5. د. وليد حمدي الأعظمي، العلاقات السعودية الأمريكية وأمن الخليج "في وثائق

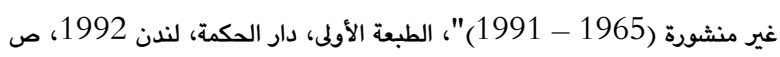

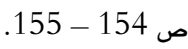

6. للمزيد من المعلومات حول هذا الموضوع أنظر: تييري كوفيل، إيران ثروة

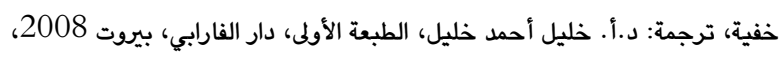

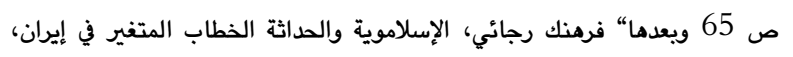

يزيد عن (950) موظفاً أمريكياً، كانوا يعملون في المؤسسات الحكومية في عهد النظام الإيراني السابق، وعينت ما يقارب (700) موظف مونف منهم

في شركة "آرامكو" وهي شركة نفطية سعودية - أمريكية (53). وأثناء إنعقاد مؤتمر القمة العربي العاشر في تونس في تشرين الثاني 1979، حاولت الحكومة الإيرانية الجديدة إرسال وفد إيراني للمشاركة

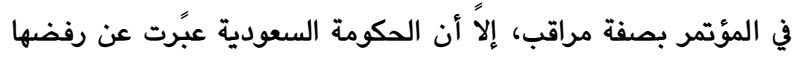
القاطع لحضور الوفد الإيراني للمؤتمر(54). ويدورها أتهمت الحكومة

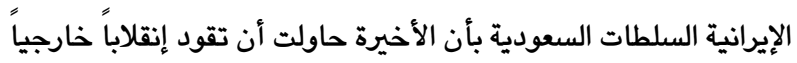

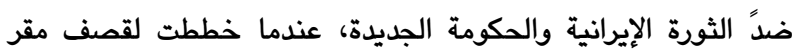
الإمام الخميني عن طريق الطيران الحربي بقيادة الطيار الإيراني السابق "ناصر ركني"، الذي إعترف لمحاولته هذه، وحصوله على مساعدات

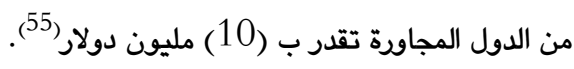
دفعت الثورة الإيرانية ببعض الجماعات الشيعية إلى التعبير عن سخطها بشكل عنيف، خلال عامي 1979 و و1980، في كل من البحرين ومدينة القطيف السعودية. وإلى جانب إرتباطها بحالة السخط التي كان تسيطر على الشيعة المحليين، فقد جاءت هذه الأحداث نتيجة لأنشطة بعض القيادات المتطرفة في نظام الثورة الإيرانية، مدفوعة بتطلعات تصدير الثورة الإسلامية خلال بواكير عهدما إلى دول الجوار الجغرافي(56).

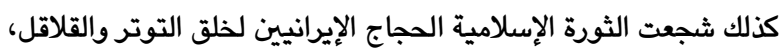
من منطلق التقسيم المذهبي "سني/شيعي"، فإن تلك الحوادث كانت سمة طبيعية للعلاقات السعودية - الإيرانية في فترة ما بعد الثورة الإيرانية عام 1979، بل وكانت الأزمات اللاحقة للتاريخ السابق أكثر فئرة خطورة من سابقتها، كونها لا تأتي من حافز المذهبي وحسب، وإنما

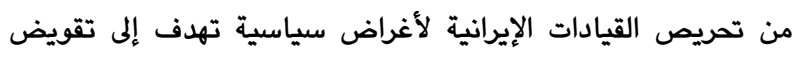
سلطة وسيادة السعودية على الأماكن المقدسة الإسلامية في المكة المكرمة والمدينة المنورة، وإلى تشكيل عوامل ضغط سياسية على القيادة السعودية لإرغامها على إثباع سياسات إقليمية ودولية تصب في مصلحة الثورة الإسلامية في إيران(57). وقد عبرت السعودية عن موقفها الرافض لهذا السلوك، وحذرت من

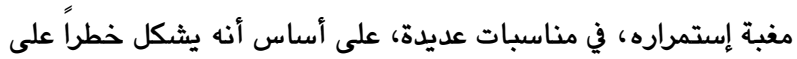
الإسلام والمسلمين في كافة أرجاء العالم الإسلامي (58).

\section{6. الخاتمة}

حاولت المملكة العربية السعودية أن تتبنى موقفاً محايداً آزاء تطور الأحداث الداخلية في إيران، خلال العام 1978 ويداية العام 1979 1979، والعمل على عدم التدخل في الشؤون الداخلية فيها، مع إعلان تأييدما لشرعية نظام محمد رضا البهلوي، الذي إستمر حتى مغادرة الأخير للبلاد في 16 كانون الثاني 1979. ويعد إنهيار النظام السابق وعودة آية الله الخميني إلى طهران في شباط

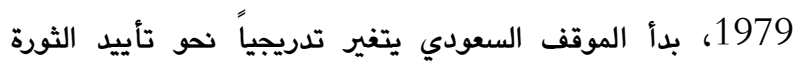


عاد إلى طهران عام 1946 أنضم إلى المعارضة عام 1953، سجن 6 سنوات أيام حكم الشاه، تولى منصب السكرتير العام لحزب إيران، أصبح أحد أعضاء اللجنة التنفيذية في الجبهة الوطنية الثالثة التي تشكلت عام 1978 بزعامة كريم سنجابي. للمزيد من المعلومات أنظر: أ.م. د. جاسم محمد هايس، "حكومة شاهبور بختيار

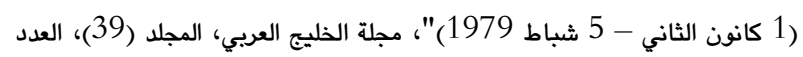

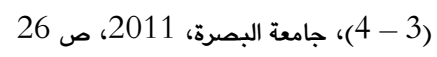
17. بنسون لي جريسون، العلاقات السعودية الأمريكية في البدء كان النفط، ترجمة: سعد هجرس، دار سينا للنشر، القاهرة 1991، ص 140 . 140. 18. إحسان نراغي، من بلاط الشاه إلى سجون الثورة، ترجمة: دار الساقي، بيروت، 1993، ص 21" د.سعيد الصباغ، العلاقات المصرية الإيرانية بين الوصال والقطيعة

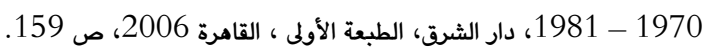
19. د.عبدالحكيم الطحاوي، المصدر السابق، ص 158. 20. مهدي بازركان: من مواليد عام 1905، درس العلوم الهندسية وتولى عام 1952 رئاسة اللجنة التنفيذية لتأميم النفط في عهد حكومة الدكتور محمد مصدق، في عام 1960 شكل حزب حركة تحرير إيران، وفي عام 1963 تم القبض عليه بتهمة التحريض على النظام الملكي، فقضى بعض سنين في المعتقل، ويعد نجاح الثورة

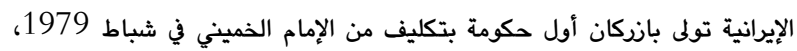
ونتيجة تسلط قوة الإمام الخميني على الأمور في البلاد، قدم بازركان إستقالته في نهاية

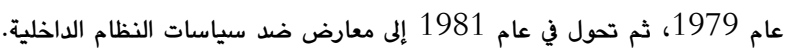
أنظر: محمد وصفي أبو مغلي، دليل الشخصيات الإيرانية المعاصرة، شعبة الدراسات الفارسية، سلسلة إيران والخليج العربي (16)، منشورات مركز دراسات الخليج العربي، جامعة البصرة 1983، ص ص 25 - 26.

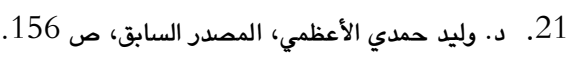

22. . د. عبدالحكيم الطحاوي، المصدر السابق، ص 158. 23. د. محمد عبدالرحمن يونس العبيدي، "سياسة إيران الخارجية تجاه إسرائيل 1979 - 2009"، مجلة دراسات إقليمية، مركز الدراسات الأقليمية، جامعة الموصل، العدد (10)، 2010، ص 4. 24. د. عبدالحكيم الطحاوي، المصدر السابق، ص ص 158 - 159. 25. نقلاً عن: بيان وكالة الأنباء السعودية، بتاريخ 3 إبريل 1979، 19 مأخوذة من صن

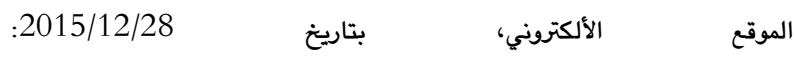
http://www.kingkhalid.org.sa/Gallery/Text/DisplayNews .aspx?View $=$ Page $\&$ news $\&$. 26. نقلاً عن: بيان وكالة الأنباء السعودية، بتاريخ 1 يوليه 1979. مأخوذة من

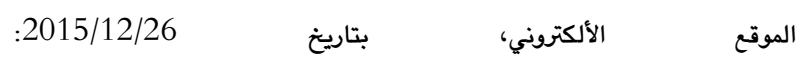
http://www.kingkhalid.org.sa/Gallery/Text/DisplayNews .aspx?View=Page \&news .

27. Ramazani، R: OP. Cit، P. 118.

28. إهتمت إيران فعلياً بمبدأ تصدير الثورة ولاسيما الى دول الخليج العربي، لأن الذليج هو المعبر الرئيسي لنفط إيران الذي يشكل العمود الفقري لإقتصاد البلاد،

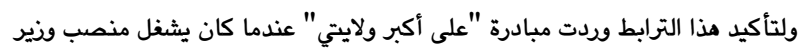

الطبعة الأولى، مركز الإمارات للدراسات والبحوث الأستراتجية - أبوظبي، 2010،

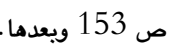
7. محمد سالم أحمد الكواز، العلاقات الإيرانية - السعودية (1979 - 2001) دراسة سياسية، مجلة دراسات إقليمية، جامعة الموصل، السنة (4)، العدد (7)،

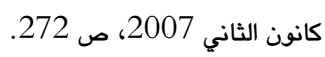
8. (8) نقلاً عن: صحيفة "الأخبار" المصرية، العدد الصادر بالتاريخ 4 يناير 1979" نقلاً عن صحيفة "عكاظ" السعودية. مأخوذة من الموقع الألكتروني، بتاريخ :2015/12/15

http://www.kingkhalid.org.sa/Gallery/Text/Disp . 9. الأزمة الإيرانية وإنعكاساتها الدولية، إعداد: مركز الدراسات السياسية والإستراتيجية، مجلة السياسة الدولية، العدد (55)، القاهرة،كانون الثاني 1979، الإنيرانيه ص 23. 10. نقلاً عن: حكام الجزيرة العربية دمي الشيطان الأكبر، مجموعة وثائق السفارة الأمريكية في طهران سلسلة مركز الجاسوسية رقم (35)، وثيقة بعنوان: من السفارة الأمريكية في جدة إلى وذارة الخارجية في واشنطن بتاريخ 3 كانون الثاني 1979، الموضوع: وجهة نظر السعودية إلى إيران، منشورات الوكالة الدولية، الطبعة الأولى،

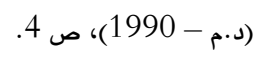
11. نقلاً عن: صحيفة "الجمهورية" المصرية، العدد الصادر بتاريخ 7 يناير 1979" نقلاً عن صحيفة "الجزيرة" السعودية. مأخوذة من الموقع الألكتروني، بتاريخ :2015/12/15

.http://www.kingkhalid.org.sa/Gallery/Text/Displ 12. نقلاً عن: صحيفة "الجمهورية" المصرية، العدد الصادر بتاريخ 21 يناير 1979. مأخوذة من الموقع الألكتروني، بتاريخ 2015/12/15: http://www.kingkhalid.org.sa/Gallery/Text/DisplayNews .aspx?View .

13. Ramazani، R: Revolutionary Iran، Challenge and Rosponse in the Middle East، "John Hopkins" Uni. Press"، London 1988، P. $116-117$.

14. للمزيد من المعلومات حول هذا الموضوع، أنظر: روبرت دريغوس، لعبة الشيطان دود الولايات المتحدة في نشأة التطرف الإسلامي، ترجمة: أشرف رفيق،

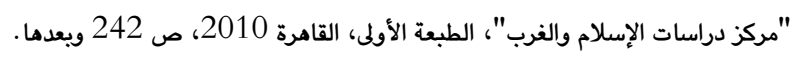
15. أيدت الإستخبارات الأمريكية والبريطانية لرئيس الوزراء القوى المعادية الايراني مصدق، ودعمتها قي تنظيم الإحتجاجات المناهضة، ويعد حين انضم الجيش الى الحركة المناهضة لرئيس الوزراء وأسقط الحكومة. وساهم التدخل الأمريكي في تقوية مواقع الشاه محمد رضا بهلوي الذي كان قد فر من إيران بعد الصراع مع مصدق،

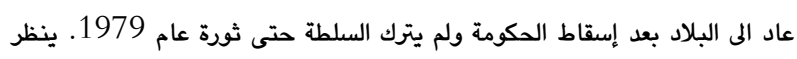

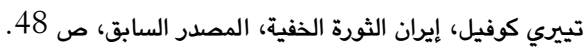
16. شاهبور بختيار: ولد عام 1916 في مدينة شهركرد، ينتمي إلى قبيلة البختيارية المعروفة في إيران، تلقى علومه في كل من لبنان وفرنسا وحصل على شهادة البكالوريوس في الفلسفة من جامعة لويس دودجر، والقانون من جامعة السوريون، ثم 
42. منصور حسن العتيبي، السياسة الإيرانية تجاه دول مجلس التعاون الخليجي

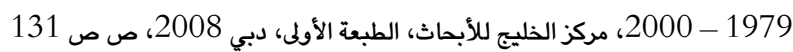

43. أنظر تقرير: صديفة "الأخبار" المصرية، العدد الصادر بتاريخ 22 نوفمبر 1979. مأخوذة من الموقع الألكتروني بتاريخ 2015/12/30: http://www.kingkhalid.org.sa/Gallery/Text/DisplayNews .aspx?View=P .

44. فيما يخص قضية الرهائن في إيران، أكدت الحكومة الإيرانية بأن الولايات المتحدة تتدخل بشؤونها الداخلية، ونتيجة لذلك قام الطلاب الإيرانيين في 4 تشرين الثاني عام 1979 بإحتجاز أثنين وخمسين رهينة من طاقم السفارة الأمريكية لمدة أربعة عشرة شهرا بطهران. وقد أعلنت إيران شروطها حول الإفراج عن الرهائن والتي تضمنت: عودة الشاه الى إيران، وإعادة ثروة الشاه، وإعتراف الولايات المتحدة وتعهدها بعدم التدخل في سياستها الداخلية والخارجية في المستقبل. للمزيد من المعلومات أنظر: أ.د. أحمد نوري ألنعيمي، "السياسة الخارجية الإيرانية تجاه الولايات المتحدة 1979 - 2008"، مجلة العلوم السياسية - جامعة بغداد، العدد (36) 45. أنظر: إحسان نراغي، المصدر السابق، ص 297. 46. منصور حسن العتيبي، المصدر السابق، ص 137. 47. عطا الله زايد الزايد، العلاقات السياسية السعودية الإيرانية وأثرها على الأمن الأقليمي لمنطقة الخليج العربي 1980 - 2003، الطبعة الأولى، "الأكاديميون

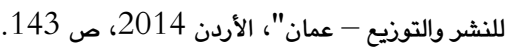

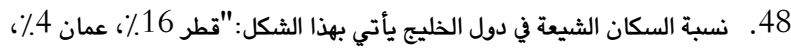
الإمارت العربية المتحدة 18٪، الكويت 24٪، البحرين 70٪، المملكة العربية السعودية 8\%، العراق 60\%". أنظر: د. رافد أحمد أمين العاني، الأمن الأقليمي الخليجي بين الخطر الإيراني والتحديات الداخلية، مجلة جامعة تكريت للعلوم القانونية والسياسية، العدد (1)، آذار 2009، صي 218.

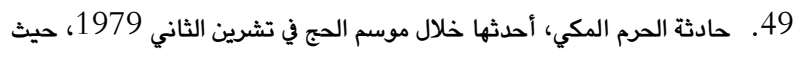
إستولى أكثر من 200 مسلح على الحرم المكي بقيادة جيهمان بن محمد العتيبي ومحمد بن عبدالله القحطاني، وأعلن أن زعيم الحركة الروحي "القحطاني" هو "المهدي المنتظر"، مطالباً بخلع الأسرة الملكية. وتفيد تقارير أخرى أن عددمم كان بين 400 و 500 مسلح. وتدخلت الحكومة السعودية مدعومة بفتوى العلماء وأنهت الحصار بعد قتل "القعطاني" مع 170 من أتباعه، وأستمر التمرد داخل الحرم لمدة إسبوعين، بعد أن قتلت مجموعة كبيرة من المهاجمين، وأسر 63 شخص منهم تم إعدامهم في 23 كانون الأول 1979 وتم القضاء على المتمردين بمساعدة قوات أردنية وعدد من المستشارين الأمريكان والفرنسين . للمزيد من المعلومات ينظر: رويرت ليسي، المملكة من الداخل "تاريخ السعودية الحديث - الملوك - المؤسسة الدينية - الليبراليون والمتطرفون"، ترجمة: خالد بن عبدالرحمن العوض، الطبعة الرابعة،

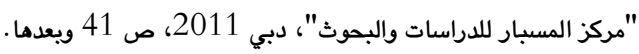
50. تدخلات أمريكا في البلدان الإسلامية، سلسلة وثائق وكر الجاسوسية الأمريكية

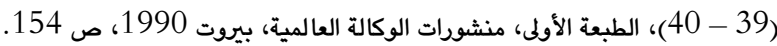

خارجية إيران بين عامي (1981 - 1997)، أذ قال "أن ساحلنا الجنوبي والخليج ومضيق هرمز وعجمان هي حدودنا الإستراتيجية الأكثر أهمية، أن هذه المنطقة حيوية بالنسبة إلينا لا يمكن أن نكون لا مبالين حيالها". للمزيد من المعلومات أنظر: فهد مزبان خزار الخزار، الجمهورية الإسلامية الإيرانية ومشروع الشرق الأوسط الكبير "رؤية

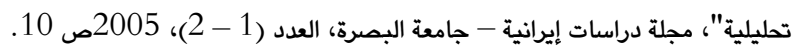
29. لمزيد من المعلومات حول هذا الموضوع أنظر تقرير: صحيفة "الأهرام" المصرية، العدد الصادر بتاريخ 13 سبتمبر 1979، مأخوذة من الموقع الألكتروني، 2015/12/29

بتاريخ .http://www.kingkhalid.org.sa/SearchHit.aspx 30. أنظر: صحيفة "الجزيرة" السعودية، العدد الصادر بتاريخ 27 يونيه 1979،

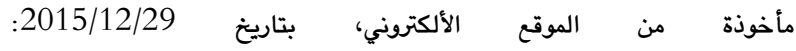
http://www.kingkhalid.org.sa/Gallery/Text/DisplayNews .aspx? View $=\mathrm{Pa}$. 31. نقلاً عن: صحيفة "الجزيرة" السعودية، العدد نفسه.

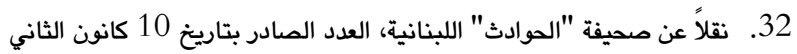
1980. مأخوذة من الموقع الألكتروني، بتاريخ 2015/12/26: http://www.kingkhalid.org.sa/SearchHit.aspx?RecID=12

$594 \&$ Search . 33. صحيفة "الجمهورية" المصرية، العدد الصادر بتاريخ 6 أغسطس 1979،

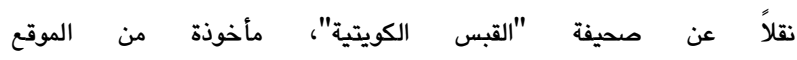
الألكتروني،بتاريخ 2015/12/29: http://www.kingkhalid.org.sa/Gallery/Text/Display . 34. صحيفة "المساء" المصرية، العدد الصادر بتاريخ 7 أغسطس 1979، نقلاً عن صحيفة "القبس الكويتية"، مأخوذة من الموقع الألكتوني، بتاريخ :2015/12/29 http://www.kingkhalid.org.sa/Gallery/Text/DisplayNew.

35. حكام الجزيرة العربية دمي الشيطان الأكبر، المصدر السابق، ص 69.

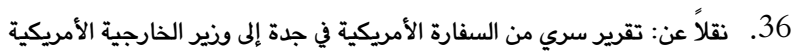
بتاريخ 10 تشرين الأول 1979، مأخوذة من: حكام الجزيرة العربية دمي الشيطان الأكبر، مجموعة وثائق....، المصدر السابق، ص 73. 37. المصدر نفسه، ص 74. 38. المصدر نفسه، ص 74.

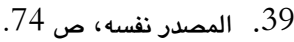
40. للمزيد من المعلومات أنظر: علي محمد حسين العامري، الإلتقاء والإفتراق في العلاقات السعودية - الأمريكية "دراسة في تطور العلاقات بين الطرفين وما آلت إليه بعد أحداث أيلول 2001"، المجلة السياسية والدولية، الجامعة المستنصرية، العدد (10)، بغداد 2008، ص 180. 41. د. وليد حمدي الأعظمي، المصدر السابق، ص صد 157. 
عثمان، ناضم يونس و شفيق ، نزار زهـير / مجلة العلوم الانسانية لجامعة زاخو، مجلد:6 ، العدد:2 ، ص 485- 494، حزيران- 2018.

55. نقلاً عن: صحيفة "الهدف" الكويتية، العدد الصادر بتاريخ 4 كانون الأول

56. بول أرتس، غيرد نونمان، وآخرون، المملكة العربية السعودية في الميزان "الإقتصاد السياسي والمجتمع والشوؤن الخارجية"، الطبعة الثانية، مركز دراسات ورنيات الوحدة العربية، بيروت 2013، ص 300. 57. عطا الله زايد الزايد، المصدر السابق، ص صدرو 147.

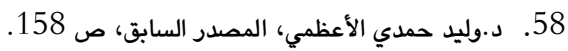

51. د. دممد سالم أحمد الكواز، المصدر السابق، ص 29.

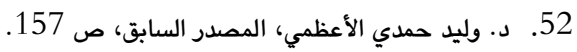

53. د. محمد سالم الكواز، العلاقات السعودية - الإيرانية 1979 - 2011 "دراسة تاريخية سياسية"، الطبعة الأولى، دار غيداء للنشر والتوزيع، عمان 2014،

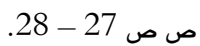
54. نقلاً عن: صحيفة "الهدف" الكويتية، العدد الصادر بتاريخ 4 كانون الأول .1979 


\title{
هلويستى شاهنشينا عهربيا سعودى ههمبهرى بهريابوونا شورهشا ئيسلاميا ثيرانى 1979
}

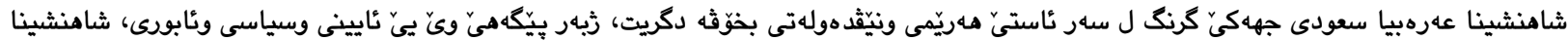

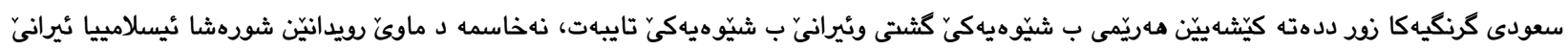

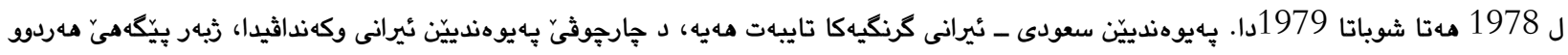

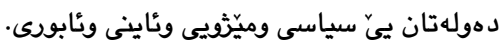

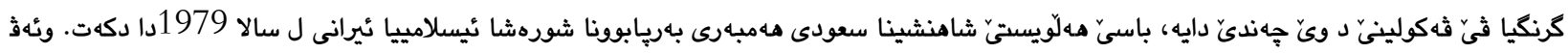

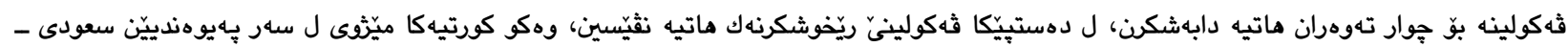

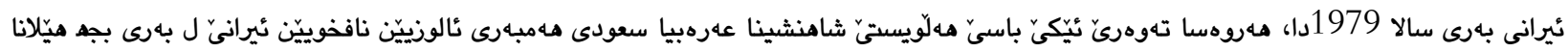

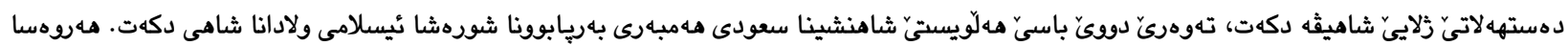

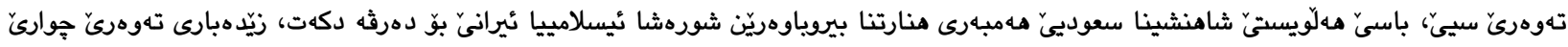

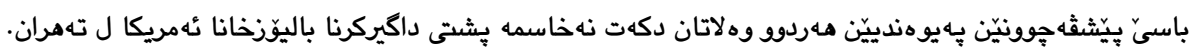

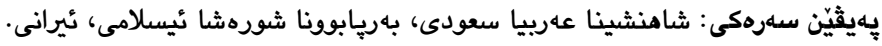

\section{Saudi Arabia's Position About the Iranian Islamic Revolution In 1979}

\begin{abstract}
:
Saudi Arabia occupied regionally and internationally prominent status since it was characterized by religious 'political and economic power. Saudi Arabia paid serious attention to the regional issues in general and to Iran in particular ، especially during the internal events in Iran in 1978 and the Iranian revolution in 1979. The Saudi - Iranian relations have gained a special importance in forming the Iranian relations system with the Gulf countries and that is due to the political 'historical rreligious and economic power that the two countries represent. The significance of the research is distinguished in shedding light on the Saudi's attitude of the Iranian Islamic revolution in 1979. The research is divided into four parts, the introduction of the research sets the ground for having a look at the Saudi - Iranian relations before 1979. The first part of the research highlights the attitude of Saudi Arabia of the internal events in Iran before the fall of the Shah. The second part is allocated for Saudi Arabia's attitude of the Iranian revolution. The third part investigates the attitude of Saudi Arabia of the principle of exporting the revolution to abroad. And the fourth part concentrated on the development of the relations between the two countries after the occupation of the American embassy in Tehran.
\end{abstract}

Keyword: Saudi Arabia's, Iranian Islamic Revolution. 